PROCEEDINGS OF THE AMERICAN MATHEMATICAL SOCIETY

Volume 124, Number 11, November 1996

\title{
ON THE COMMUTANT OF HYPONORMAL OPERATORS
}

\author{
BEBE PRUNARU \\ (Communicated by Palle E. T. Jorgensen)
}

\begin{abstract}
Let $T$ be a pure hyponormal operator with compact self-commutator. We show that the unit ball of the commutant of $T^{*}$ is compact in the strong operator topology.
\end{abstract}

Let $H$ be a separable complex Hilbert space and let $L(H)$ denote the algebra of all bounded linear operators on $H$. An operator $T \in L(H)$ is hyponormal if $T^{*} T-T T^{*} \geq 0$ and essentially normal if $T^{*} T-T T^{*}$ is compact. Moreover $T$ is pure if it has no nonzero reducing subspace on which it is normal. The main result of this paper is the following:

Theorem 1. Let $T \in L(H)$ be a pure hyponormal essentially normal operator. Then the closed unit ball of the commutant algebra $\left\{T^{*}\right\}^{\prime}$ is compact in the strong operator topology.

The proof of this theorem relies on the following two results. The first one is a particular case of a more general result obtained by $\mathrm{V}$. Lomonosov.

Theorem 2 (cf. [L, Lemma 1]). Let $T \in L(H)$ be an essentially normal operator. Suppose there exists a sequence $\left\{T_{n}\right\}$ in the commutant of $T$ such that $T_{n} \rightarrow 0$ weakly but $T_{n} \nrightarrow 0$ strongly. Then there exist a normal operator $N \in L(H)$ and a nonzero operator $X \in L(H)$ such that $N X=X T$.

This theorem has been used in $[\mathrm{L}]$ to show that for a transitive essentially normal operator $T \in L(H)$ either $\{T\}^{\prime}$ or $\left\{T^{*}\right\}^{\prime}$ has strongly compact ball. The second result we need is an asymmetric Fuglede-Putnam theorem due to J.G. Stampfli and B.L. Wadhwa.

Theorem 3 (cf. [SW, Theorem 1]). Let $T \in L(H)$ be a hyponormal operator and suppose there exist a normal operator $N \in L(H)$ and $X \in L(H)$ with dense range, such that $T X=X N$. Then $T$ is normal.

Proof of Theorem 1. Suppose the conclusion is false. Now, recall that the strong topology is metrizable on bounded subsets of $L(H)$ and that the unit ball of $L(H)$ is metrizable and compact in the weak operator topology. Therefore there exists a sequence of operators $\left\{T_{n}\right\}$ in $\left\{T^{*}\right\}^{\prime}$ such that $T_{n} \rightarrow 0$ weakly but $T_{n} \nrightarrow 0$ strongly. An application of Theorem 2 above yields a normal operator $N \in L(H)$ and a nonzero operator $X \in L(H)$ such that $N X=X T^{*}$. Therefore $T X^{*}=X^{*} N^{*}$

Received by the editors May 8, 1995.

1991 Mathematics Subject Classification. Primary 47B20; Secondary 47D25.

Key words and phrases. Hyponormal operators, strong operator topology, operator algebras, commutant.

(C)1996 American Mathematical Society 
and the restriction $S$ of $T$ to $\overline{\operatorname{ImX} X^{*}}$ is still hyponormal. Applying Theorem 3 we infer that $S$ is normal, which contradicts our initial assumption that $T$ is pure hyponormal. The proof is complete.

The following is an easy consequence of Theorem 1.

Corollary 1. Under the assumptions of Theorem 1, the weak*, weak and strong operator topologies agree on bounded subsets of $\left\{T^{*}\right\}^{\prime}$.

For a compact set $K \subset C$ let $\operatorname{Rat}(K)$ denote the algebra of all rational functions with poles off $K$. An operator $T \in L(H)$ is multicyclic if there exists a finite family of vectors $\left\{x_{1}, \ldots, x_{m}\right\}$ in $H$ such that the space $\left\{\sum_{k=1}^{m} f_{k}(T) x_{k} ; f_{1}, \ldots, f_{m} \in\right.$ $\operatorname{Rat}(\sigma(T))\}$ is dense in $H$.

Corollary 2. Let $T \in L(H)$ be a multicyclic pure hyponormal operator. Then the weak*, weak and strong operator topologies agree on bounded subsets of $\left\{T^{*}\right\}^{\prime}$.

Proof. Since $T$ is multicyclic hyponormal, it is also essentially normal by a celebrated theorem of Berger and Shaw [BS]. Now, an application of Theorem 1 yields the conclusion.

\section{REFERENCES}

[BS] C.A. Berger and B.I. Shaw, Selfcommutators of multicyclic hyponormal operators are always trace-class, Bull. Amer. Math. Soc. 79 (1973), 1193-1199. MR 51:11168

[L] V. Lomonosov, A construction of an intertwining operator, Funktsional. Anal. i Prilozhen. 14 (1980), 67-68; English transl. in Functional Anal. Appl. 14 (1980), 54-55. MR 81k:47032

[SW] J.G. Stampfli and B.L. Wadhwa, An asymmetric Putnam-Fuglede theorem for dominant operators, Indiana Univ. Math. J. 25 (1976), 359-365. MR 53:14197

Department of Mathematics, Indiana University, Bloomington, Indiana 47405

E-mail address: bprunaru@ucs.indiana.edu

Institute of Mathematics, Romanian Academy, P.O.Box 1-764, 70700 Bucharest, RoMANIA 